\title{
Evaluación de la estructura del ensamble de peces de un arrecife costero norpatagónico sometido a diversos impactos antrópicos
}

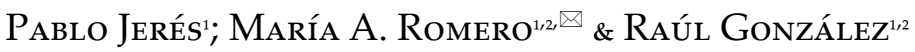 \\ ${ }^{1}$ Escuela Superior de Ciencias Marinas, Universidad Nacional del Comahue, Argentina. ${ }^{2}$ CONICET, Argentina.
}

\begin{abstract}
Resumen. En comparación con los arrecifes de regiones tropicales, los arrecifes rocosos de la costa patagónica se caracterizan por una menor riqueza específica, pero una mayor biomasa y proporción de peces de gran tamaño. Estos arrecifes son afectados por cambios estacionales notables que impactan sobre la abundancia y la diversidad de peces. Considerando la escasez de estudios realizados sobre estos ecosistemas en la región norpatagónica, el objetivo del presente trabajo fue evaluar los cambios potenciales en la estructura del ensamble de peces en un arrecife rocoso del Golfo San Matías tras más de 20 años de estar sujeto a diversos impactos antrópicos y a cambios ambientales. Además, se evaluó la variación del ensamble y su relación con las características ambientales a lo largo de un ciclo anual. Para esto, se realizó un muestreo mediante buceo autónomo en el Parque Submarino Las Grutas. Se identificó el mismo conjunto íctico que el descripto previamente en base a datos de 1990, conformado por cinco especies templado-cálidas -Diplodus argenteus, Pinguipes brasilianus, Acanthistius patachonicus, Serranus auriga y Pseudopercis semifasciata - y un único registro de una especie templado-fría, Congiopodus peruvianus. Sin embargo, se identificó un aumento de la abundancia para la totalidad de las especies; incluso, algunas como $D$. argenteus y $S$. auriga duplicaron y triplicaron, respectivamente, su abundancia respecto a los valores de 1990. Este aumento podría deberse a cambios ambientales (e.g., de la producción primaria o de la temperatura del mar) que favorecerían el aumento de las poblaciones de peces o el desplazamiento de peces desde poblaciones cercanas. Con este trabajo destacamos la importancia de realizar monitoreos a largo plazo a fin de evaluar el estado de los ecosistemas costeros y establecer medidas de manejo y conservación que preserven su estructura y funcionamiento a lo largo del tiempo.
\end{abstract}

[Palabras clave: diversidad, impacto humano, ecosistemas de la costa]

Aвstract. Assessment of the structure of fish assembly of a North-Patagonian coastal reef subjected to different anthropic impacts. The rocky reefs of the Patagonian coast are characterized by lower specific richness, but higher biomass and proportion of large fish than reefs in tropical regions. Rocky reefs have major seasonal changes that can affect fish abundance and diversity. Ecological studies on the North-Patagonian reef ecosystems are scarce. The aim of the present study was to evaluate the potential changes in the fish assemblage composition of a rocky reef in the San Matías Gulf. An abundance reduction of fish species is expected after more than 20 years of anthropogenic impacts. The assemblage variation and its relationship with environmental traits were evaluated over one year. Fish species abundance was recorded using scuba diving in the Parque Submarino Las Grutas. The newly obtained results were compared to a previous study by González (1993). The results showed that the fish assemblage composition is identical to that in the 1990s, consisting of five warm-tempered species (Diplodus argenteus, Pinguipes brasilianus, Acanthistius patachonicus, Serranus auriga and Pseudopercis semifasciata) and a single record of a cold-tempered species (Congiopodus peruvianus). However, in terms of abundance, an increase was observed for all species. Species such as D. argenteus and S. auriga nearly doubled and tripled their abundance, respectively, compared to the 1990 levels. Environmental factors such as sea temperature rise and primary production upturn could promote an increase in the fish population numbers and/or the migration of fish from nearby populations. Throughout the year, the species showed a tendency coupled with temperature, giving that all the species showed greater abundances at the end of the warm season (April). In this study, we demonstrate the need of long-tern ecological studies in order to evaluate the condition of our coastal ecosystems to help establish management and conservation measures that preserve them over time.

[Keywords: diversity, human impacts, coastal ecosystems]

Editora asociada: Irina Izaguirre

romero.ale@gmail.com
Recibido: 10 de agosto de 2017

Aceptado: 16 de enero de 2018 


\section{INTRODUCCIÓN}

Los ecosistemas marinos del mundo se encuentran expuestos a diversas amenazas que conducen a la pérdida de diversidad y de abundancia biológica. En gran medida, esta pérdida de biodiversidad es consecuencia de las actividades humanas, ya sea de manera directa (sobrexplotación) o indirecta (alteración del hábitat) (Moreno 2001). Algunos de los factores que pueden ocasionar cambios en la estructura de los ecosistemas impactados son la contaminación de origen antrópico y la eutrofización del medio marino (Josefson et al. 2008; Johnston and Roberts 2009), la sobrepesca (Aubone 2000; Jackson et al. 2001; Kaiser and De Groot 2001), las fluctuaciones de las variables ambientales (Stige et al. 2006; Johnson et al. 2011) y la introducción de especies exóticas (Hollebone and Hay 2007).

En particular, los ambientes costeros recibieron un impacto creciente debido a la tendencia a incrementar las poblaciones en las zonas costeras (Dias et al. 2012). En la actualidad, vive más gente en la franja costera a menos de $100 \mathrm{~km}$ del mar que la que vivía en todo el planeta en 1950 (Castro and Huber 2007). Por ejemplo, la población del balneario Las Grutas, Río Negro, ha crecido de 760 habitantes permanentes en el año 1991 a 4807 en el año 2010 (INDEC 1991, 2010). Lo mismo ocurre con el turismo en la región, que crece entre $15 \%$ y $25 \%$ año tras año. Este constante crecimiento de las poblaciones costeras y de la cantidad de turistas que eligen vacacionar en las playas lleva a que los efectos antrópicos que impactan sobre los ecosistemas costeros marinos sean cada vez mayores.

Cualquier estrategia de protección del medio natural debe asegurar la conservación de la funcionalidad de los ecosistemas. Un punto clave a la hora de definir estrategias de manejo es poseer herramientas fiables capaces de medir la variación de la estructura de las comunidades en el espacio y en el tiempo (Moreno 2001). Para ello, es necesario efectuar estudios ecológicos y monitoreos regulares en los ecosistemas a fin de establecer medidas de manejo y conservación adecuadas. En nuestro país, la dificultad general de acceso a sitios de estudio en la costa y el mar, la falta de recursos y el desinterés produjo una falta de programas de monitoreo a largo plazo y de series temporales de datos biológicos, ecológicos y ambientales de estos sistemas. Estos datos son fundamentales para comprender la dinámica de las poblaciones, para rastrear cambios en los procesos biológicos, físicos y químicos y para detectar y estimar las alteraciones que el ser humano produjo a lo largo del tiempo.

Los arrecifes rocosos representan uno de los hábitats más característicos de la zona costera de los golfos norpatagónicos. Los ensambles de peces asociados a estos arrecifes fueron descriptos como sistemas con una baja diversidad conformados por varias especies longevas como Pseudopercis semifasciata (salmón de mar) y Acanthistius patachonicus (mero) (González 1993; Galván 2008; Galván et al. 2009a). Además, estas especies presentan un crecimiento lento, una época de reproducción extensa con desoves múltiples y una recuperación lenta del agotamiento somático (Elías and Rajoy 1992; González 1998; Rubinich 2000), lo cual las hace muy susceptibles al impacto antrópico (e.g., el incremento en la mortalidad debido a la pesca) (Sadovy and Cheung 2003).

No obstante, a pesar de la importancia biológica y de los impactos antrópicos producto de los intereses socioeconómicos que existen sobre la región, los ensambles de peces asociados a los arrecifes rocosos del Golfo San Matías (GSM) permanecen poco estudiados. El único antecedente disponible para el área noroeste del GSM es el estudio de González (1993), quien evaluó la variación en la abundancia mensual de peces en un arrecife rocoso, sobre la base de datos tomados en el año 1990. Los arrecifes y los peces de los golfos norpatagónicos sustentaron actividades de pesca deportiva (submarina y de altura) y de buceo recreativo desde la década del cincuenta (Sanabra 2002). A escala local, el área considerada en este estudio también recibió un impacto antrópico creciente a causa de su cercanía al centro turístico Las Grutas (Scalise et al. 2009; Kokot and Chomnalez 2012). Esto conlleva un aumento de la exposición a contaminación de origen antrópico (e.g., vertidos cloacales), con la consecuente eutrofización del medio marino (Santinelli 2008; Martinetto et al. 2010; Teichberg et al. 2010). Sumado a estos cambios, las fluctuaciones en las variables ambientales a escala global producto del cambio climático (Stige et al. 2006; Johnson et al. 2011) podrían estar afectando tanto la composición como la abundancia de las especies de peces que habitan en los arrecifes patagónicos.

Bajo el marco teórico del concepto de diversidad y teniendo en cuenta la importancia de monitorear los ecosistemas a largo plazo, 
el objetivo general del presente estudio fue evaluar los potenciales cambios en la estructura de un ensamble de peces, luego de más de 20 años de estar sujeto a diversos impactos antrópicos y cambios ambientales. Para poner a prueba la hipótesis que postula un cambio en la composición y la abundancia de los peces de arrecifes, con una tendencia hacia la reducción en la importancia de aquellas especies más sensibles a los cambios ambientales, se seleccionó como área de estudio el mismo arrecife considerado por González (1993), localizado frente al balneario Las Grutas. Se espera que la información generada permita evaluar la estabilidad de los ensambles de peces de arrecifes y su potencial como indicadores del impacto antrópico, a fin de contribuir a la conservación de los ecosistemas costeros del Mar Argentino.

\section{Materiales y Métodos}

\section{Área de estudio y caracterización de los impactos antrópicos}

El Golfo San Matías se extiende entre los $40^{\circ} 45^{\prime} \mathrm{S}-42^{\circ} 14^{\prime} \mathrm{S}$ y los $63^{\circ} 05^{\prime}$ y $65^{\circ} 10^{\prime} \mathrm{O}$. $\mathrm{Su}$ superficie aproximada es de $19700 \mathrm{~km}^{2}$. Constituye una cuenca marina semicerrada, con profundidades máximas de hasta $220 \mathrm{~m}$ en su parte central y un zócalo de profundidades relativamente menores (50$80 \mathrm{~m}$ ) en su boca (Piola and Scasso 1988). El GSM se encuentra localizado en la transición entre las dos provincias biogeográficas del Mar Argentino: la provincia Argentina, con aguas templado-cálidas y especies que se distribuyen típicamente entre Brasil y el norte de Patagonia, y la provincia Magallánica de aguas templado-frías, caracterizada por especies que se distribuyen desde el sur de la provincia de Buenos Aires hacia latitudes mayores (Menni and Stehmann 2000; Balech and Ehrlich 2008). El GSM constituye un área de relevancia biogeográfica donde coexisten especies típicas tanto de aguas cálidas como de aguas frías.

A lo largo de la historia existieron $-y$ siguen existiendo- diferentes intereses socioeconómicos (e.g., la pesca y el turismo) sobre los recursos biológicos del GSM. La pesca es uno de los principales sustentos económicos para la región. La especie blanco de la pesquería dirigida al conjunto demersal es la merluza común, Merluccius hubbsi, que en el golfo constituye una subpoblación o stock pesquero unitario, independiente de los stocks de plataforma (Di Giácomo et al. 1993; González et al. 2007). Sobre esta especie se encuentran dirigidas dos flotas pesqueras: una industrial que utiliza como arte de pesca la red de arrastre de fondo, y otra artesanal con palangre de media agua. Ambas producen la captura de un número de especies acompañantes como el mero y el salmón de mar (Romero et al. 2008).

En los últimos años, la pesca de arrastre en el Golfo San Matías registró un incremento en el esfuerzo pesquero, con volúmenes crecientes de descartes y con una tendencia a la diversificación de los desembarcos (Figura 1) (Romero et al. 2008, 2010). En particular, durante el período considerado en este estudio (1990 a 2015) se pasó de capturar 3612 t de peces en 1990 a 6617 t en 2015 (Millán 2015).

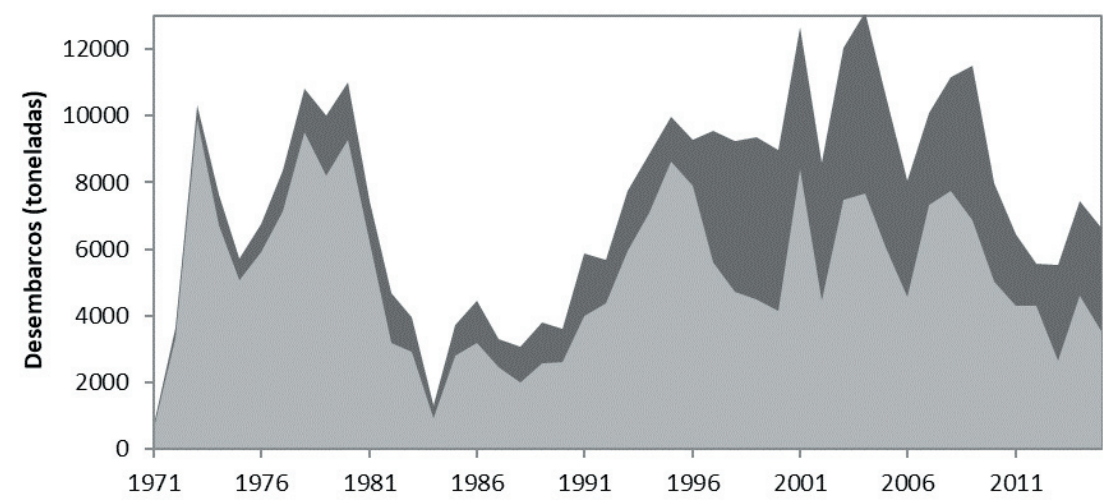

Figura 1. Desembarcos pesqueros generados por la pesca de arrastre demersal que opera en el Golfo San Matías desde el inicio de la actividad en 1971 hasta 2015. En gris oscuro se presentan los desembarcos totales y en gris claro los desembarcos de merluza común (Merluccius hubbsi). Datos obtenidos a partir de la Estadística Pesquera de la provincia de Río Negro (Millán 2015).

Figure 1. Fishing landing of the trawl demersal fishery that operates in San Matías Gulf for the period 1971-2015. Total landings are presented in dark grey, while common hake (Merluccius hubbsi) landings are shown in light grey. Data obtained from Fishery Statistics from Rio Negro province (Millán 2015) 
Además de la pesca, el golfo sostiene una actividad turística creciente enfocada en el avistaje de fauna marina. Esta actividad se desarrolla en el sector noroeste del golfo, dentro de los límites geográficos del Area Marina Protegida Bahía San Antonio (ANPBSA). En estas excursiones se pueden avistar cetáceos, pinnípedos y aves marinas. Otra actividad turística enfocada en el avistaje de fauna marina es la práctica de buceo autónomo recreativo. Esta actividad se lleva a cabo en los principales arrecifes rocosos cercanos a la playa de Las Grutas. Aunque no se dispone de datos oficiales, en los últimos años aumentó la cantidad de turistas que frecuentan la región atraídos por esta actividad, según lo mencionado por los operadores de buceo. Finalmente, la pesca deportiva sobre arrecifes rocosos a bordo de pequeñas embarcaciones es otra de las ofertas turísticas de la región y también registra una demanda creciente.

Sumado a los impactos que produce la remoción por pesca (i.e., pesca costera con caña, caza submarina, pesca artesanal y de arrastre industrial), la navegación de embarcaciones de diferente porte y la destrucción de hábitats marinos (Scalise et al. 2009), la contaminación y la eutrofización por la cercanía a los centros urbanos (San Antonio Oeste y Las Grutas) es una de las consecuencias más significativas del impacto antrópico sobre el hábitat costero. En varios puntos de la costa de la Bahía de San Antonio y las playas de Las Grutas se detectó contaminación bacteriana; en algunos sitios, los valores medidos del grupo de Enterococos se encontraron por encima del umbral establecido para sitios recreativos (Narvarte 2006; Scalise et al. 2009). Asimismo, se reportaron concentraciones elevadas de nutrientes, floraciones de macroalgas y altas tasas de crecimiento de Ulva lactuca en el área de influencia de la Bahía de San Antonio (Martinetto et al. 2010; Teichberg et al. 2010). Se sugirió que la fuente más probable de microorganismos y nutrientes que conducen a este proceso de eutrofización estaría vinculada a la descarga de efluentes líquidos desde tierra firme, probablemente por filtración a través del sedimento (Narvarte 2006; Scalise et al. 2009; Martinetto et al. 2010).

Los muestreos se llevaron a cabo sobre un sector conocido como "Parque Submarino Las Grutas" (PSLG) (4048' S - 650.' O), una formación rocosa submarina paralela a la costa, situada a 1 milla náutica de la misma, frente a la playa de Las Grutas (Figura 2). El PSLG comprende una restinga lineal con

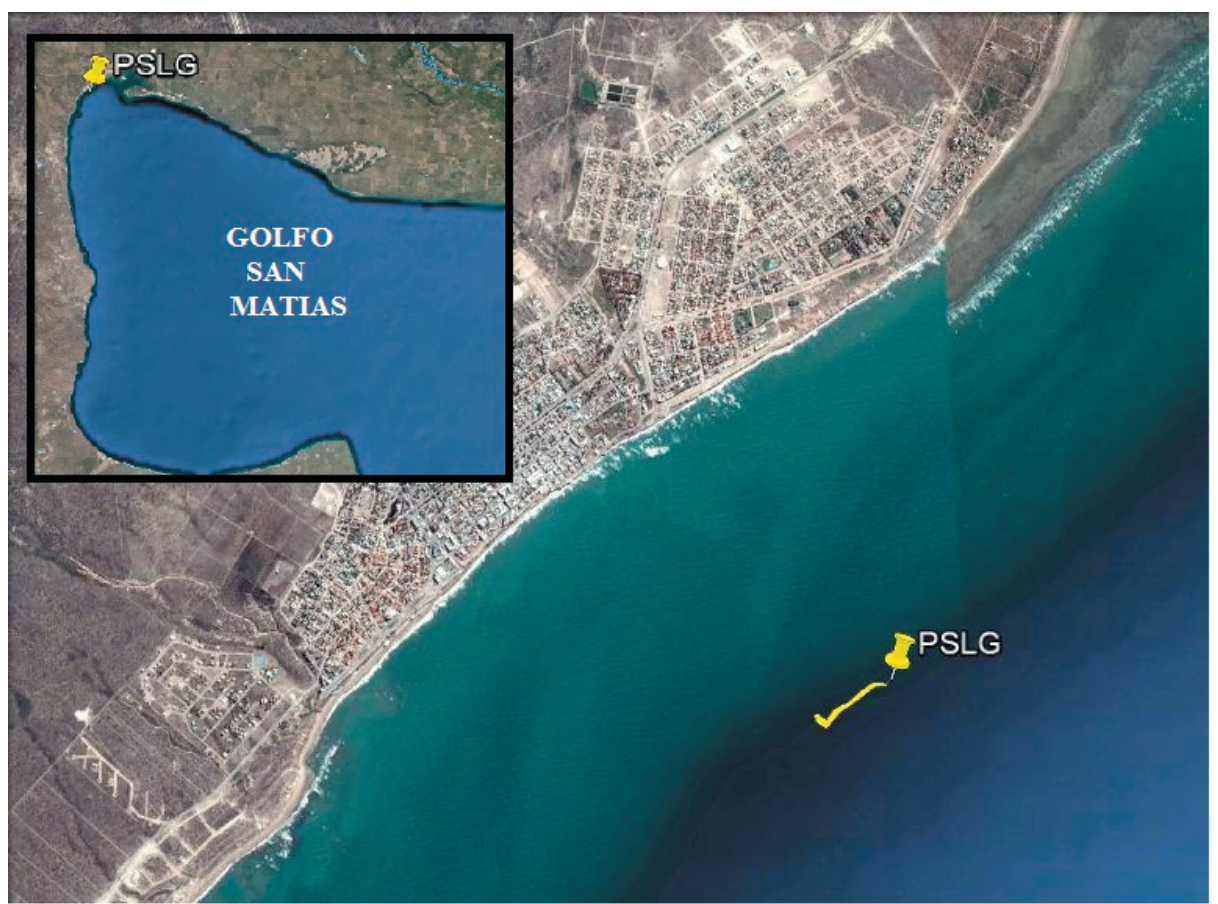

Figura 2. Ubicación del Parque Submarino Las Grutas (PSLG) dentro del Golfo San Matías. Imagen realizada en base a fotografías obtenidas de Google Earth (Versión 7.1.7.2606).

Figure 2. Location of the Parque Submarino Las Grutas (PSLG) within the San Matías Gulf. Image based on photographs obtained from Google Earth (Version 7.1.7.2606). 
profundidades de entre 4 y $13 \mathrm{~m}$, tiene una longitud de $\sim 300 \mathrm{~m}$ y un ancho variable de entre 2 y $10 \mathrm{~m}$. A lo largo de su extensión se eleva del fondo entre 0.8 y $1.7 \mathrm{~m}$ y genera una diversidad de microhábitats bien diferenciables a partir de la disposición, el tamaño y la forma de las cuevas y las rocas que la componen. Constituye la saliente submarina más notable del lugar. A su alrededor se extienden fondos llanos de arena y sedimento consolidado, y no existen otras formaciones similares a menos de 500 m (González 1993). Los muestreos se llevaron a cabo en el borde sud-oeste de la restinga, donde se genera un escalón entre las piedras que la conforman y el fondo, lo que ofrece una gran cantidad de microhábitats. Esta formación particular favorece una gran abundancia y diversidad de peces en el área. El PSLG fue muy aprovechado con fines turísticos; desde hace más de 15 años lo visitan muy a menudo los operadores de buceo debido a su belleza y a que se puede acceder con facilidad por su cercanía a la costa de Las Grutas.

\section{Recolección de los datos}

El período de muestreo abarcó desde marzo de 2015 a enero de 2016. En este lapso se llevaron a cabo siete censos submarinos en diferentes meses. Los meses faltantes se debieron a la imposibilidad de salir a muestrear como consecuencia de condiciones meteorológicas desfavorables, en su gran mayoría relacionadas con fuertes vientos del sur. Estas malas condiciones dificultaron o impidieron navegar hasta el sitio de muestreo $\mathrm{y}$, en caso de lograrlo, las observaciones submarinas se tornaron muy difíciles dada la agitación del agua y la escasa visibilidad por los sedimentos en suspensión. A fin de asegurar la consistencia en la toma de datos, todos los conteos de 2015 y 2016 los realizó el mismo observador, quien fue capacitado y entrenado por el responsable de la ejecución de los censos de 1990.

\section{Metodología de los censos visuales subacuáticos}

Los censos se realizaron mediante buceo autónomo bajo condiciones ambientales similares, seleccionando el mismo estado de marea (entre la pleamar y la bajamar), velocidad y dirección de la corriente, y momento del día (12:00 a 18:00 h). Para comparar los resultados con los obtenidos por González (1993), la metodología empleada para realizar los censos fue similar a la descripta en su estudio. En cada censo se recorrió una misma transecta de longitud y ancho constante, método conocido como "strip transect". Para ello, se delimitó una transecta de largo constante $(85 \mathrm{~m})$ en el mismo sector de la restinga donde se colectaron los datos del trabajo de González (1993), en el sector sud-oeste del PSLG (Figura 2). Se delimitó el largo de la transecta mediante dos obstáculos artificiales que ya se encontraban sumergidos en este sector del PSLG; para todos los censos se respetó este mismo largo de transecta. El ancho de la transecta $(2 W)$ fue establecido en cada oportunidad de muestreo en función de las condiciones de visibilidad. En cada inmersión, previo a comenzar a recorrer la transecta, se determinó la máxima distancia (W) hasta la cual fue posible identificar a los peces. Para esto, se seleccionó una piedra de tamaño similar al promedio del tamaño de los peces y se fue tomando distancia de la misma hasta el punto máximo en el que esta se mantuvo reconocible. Luego, se midió esta distancia y se tomó el doble de la misma (2W) como ancho constante para la transecta en ese censo. Por otro lado, se tomó la temperatura del agua en el fondo mediante una computadora de buceo autónomo (período marzo-agosto de 2015).

Se registró el número de individuos de cada especie de peces presentes a lo largo de la transecta. Para ello se recorrió la misma mediante buceo autónomo y se registró, en planillas plásticas resistentes al agua, el número de individuos por especie presentes en el cuadrante de $180^{\circ}$ por delante del observador. Cuando se encontraron cardúmenes con un número de individuos mayor a 20, se estimó el número total de individuos en base a rangos de abundancia, respetando el mismo criterio de estimación para cada una de las especies y entre los diferentes muestreos. En cada una de las salidas, el largo total de la transecta fue recorrido en dos ocasiones a fin de lograr una repetición de la estimación. Al analizar los datos se consideró el promedio obtenido a partir de los conteos generados en ambos recorridos de la transecta.

\section{Análisis de los datos}

Abundancia. Para determinar la abundancia de cada una de las especies de peces presentes en el arrecife se calcularon las densidades por especie (individuos $/ \mathrm{m}^{2}$ ) según la fórmula:

$\mathrm{D}=\mathrm{nw} /$ 2LW (Burnham et al. 1985) 
donde $\mathrm{D}=$ densidad media estimada, nw=número de peces contados en la transecta, $\mathrm{L}=$ longitud de la transecta, $\mathrm{W}=$ mitad del ancho de la transecta.

Sobre la base del promedio de los conteos de las dos transectas de cada muestreo, se calculó el número medio de especies por censo (riqueza específica), la diversidad específica (índice de Shannon-Weaver) y la uniformidad o equitatividad ("evenness") (Pielou 1975) para los siete meses relevados. Se calculó la riqueza como el número total de especies presentes en una muestra/transecta. Se utilizó el índice de Shannon-Weaver para estimar la diversidad específica, dado que permitió comparar los resultados del presente estudio con los obtenidos por González (1993). En este caso es más informativo usar el mismo índice que se utilizó antes en la misma zona, en lugar de usar otros índices que pudieran ser teórica y biológicamente más aceptables (Magurran 1988).

Para verificar la existencia de diferencias en la comunidad de peces en cuanto a la variabilidad anual (1990 vs. 2015) se empleó un análisis de varianza multivariado de una vía con base en permutaciones (PERMANOVA) (Anderson 2001). Previo al análisis, los datos fueron transformados mediante la raíz cuadrada, dada la disimilitud en la abundancia relativa de las diferentes especies, y se chequeó la homogeneidad de las varianzas entre grupos por el procedimiento de Marti Anderson's PERMDISP2 (función betadisper del paquete vegan) (Oksanen et al. 2017). El análisis se basó en la distancia de Bray-Curtis a partir de 4999 permutaciones de los residuos del modelo para obtener los p-valores. Cuando se hallaron diferencias significativas, se utilizó la técnica de análisis SIMPER (PRIMER) (Clarke 1993; Clarke and Warwick 2001) para evaluar las especies responsables de la disimilitud entre los niveles del factor. Todos los análisis estadísticos fueron ejecutados en $\mathrm{R}$ versión 3.4.2.

\section{Resultados}

A lo largo del período de estudio se detectó un total de 6 especies de peces presentes en el PSLG. Cuatro de las mismas tuvieron 100\% de ocurrencia en los muestreos: Diplodus argenteus (sargo), Pinguipes brasilianus (turco), Acanthistius patachonicus (mero) y Serranus auriga (cocherito). Pseudopercis semifasciata (salmón de mar) fue registrado en 5 de los 7 muestreos, mientras que de Congiopodus peruvianus (chanchito) sólo se detectó un individuo en el muestreo del mes de noviembre de 2015 (Tabla 1).

La riqueza específica se mantuvo estable a lo largo del año; osciló entre 4 y 6 debido a la presencia especies poco frecuentes. La diversidad registró valores entre 0.4 y 0.6 a lo largo del año. La uniformidad tomó valores entre 0.6 y 0.8 , lo cual indicó una equitatividad relativamente alta entre las especies presentes en el PSLG (Tabla 1).

En la Figura 3 se observa la densidad media por especie estimada para cada mes. Tres especies (sargo, mero y cocherito) de las cuatro más frecuentes siguieron un patrón similar, ya que su densidad disminuyó hacia

Tabla 1. Abundancia promedio (número de individuos) por especie para los siete meses muestreados en el Parque Submarino Las Grutas (PSLG). W: mitad del ancho de transecta (en metros); S: riqueza específica; H': diversidad de Shannon-Weaver; E: uniformidad. Entre paréntesis se indica el desvío estándar de la estimación.

Table 1. Mean abundance (number of individuals) per species for the seven months sampled in the Parque Submarino Las Grutas (PSLG). W: half the width of the transect (in meters); S: species richness; H': Shannon-Weaver diversity index; E: uniformity. The standard deviation of the estimate is given in brackets.

\begin{tabular}{|c|c|c|c|c|c|c|c|c|c|c|}
\hline \multirow[b]{2}{*}{ Fecha } & \multirow[b]{2}{*}{ W } & \multirow{2}{*}{$\mathrm{S}$} & \multirow[b]{2}{*}{$\mathrm{H}^{\prime}$} & \multirow[b]{2}{*}{$\mathrm{E}$} & \multicolumn{6}{|c|}{ Abundancia } \\
\hline & & & & & Sargo & Turco & Mero & Salmón & Cocherito & Chanchito \\
\hline mar-15 & 3 & 5 & 0.50 & 0.71 & $98(19.80)$ & $39(2.83)$ & $20(8.49)$ & $1(0.00)$ & 15 (7.07) & 0 \\
\hline abr-15 & 3 & 5 & 0.56 & 0.80 & $111(19.80)$ & $66(2.83)$ & $44.5(19.09)$ & $0.5(0.71)$ & $29(9.90)$ & 0 \\
\hline may-15 & 4 & 5 & 0.52 & 0.75 & 113 (14.14) & $90.5(23.33)$ & $16.5(9.19)$ & $1.5(0.71)$ & $34(2.83)$ & 0 \\
\hline jun-15 & 4 & 5 & 0.51 & 0.73 & $143(25.46)$ & $27.5(3.54)$ & $36(1.41)$ & $2.5(0.71)$ & 37 (7.07) & 0 \\
\hline ago-15 & 3.5 & 5 & 0.51 & 0.73 & 32 (11.31) & 88.5 (7.78) & $17.5(0.71)$ & $2.5(0.71)$ & $13.5(4.95)$ & 0 \\
\hline nov-15 & 4 & 5 & 0.43 & 0.61 & $88.5(41.72)$ & 38.5 (4.95) & $6.5(3.54)$ & 0 & $8(2.83)$ & $1(1.41)$ \\
\hline ene-16 & 4 & 4 & 0.49 & 0.81 & $69.5(1.061)$ & $24(5.66)$ & $23(7.07)$ & 0 & $7(2.83)$ & 0 \\
\hline
\end{tabular}




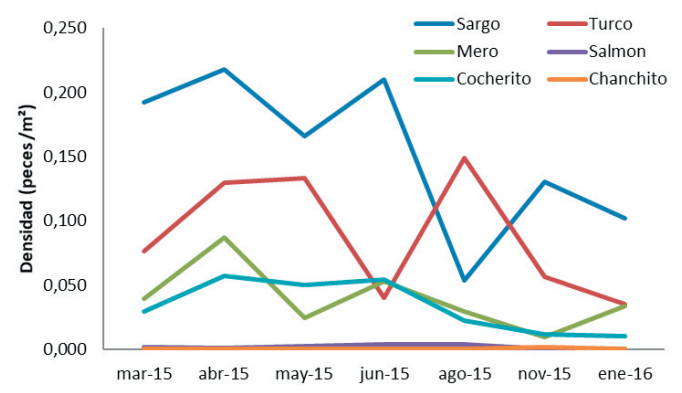

Figura 3. Densidad media estimada por $\mathrm{m}^{2}$ para cada una de las especies observadas en el Parque Submarino Las Grutas.

Figure 3. Mean estimated density per $\mathrm{m}^{2}$ for each observed species in the Parque Submarino Las Grutas.

primavera-verano y alcanzó los valores máximos durante el otoño. En particular, esto es notorio en el caso del sargo, especie para la cual se identificaron grandes cardúmenes de juveniles $(<10 \mathrm{~cm}$ de largo total) en los muestreos de enero y marzo. El turco mostró un patrón diferente, con un pico máximo de densidad en el mes de agosto y mínimos en junio y enero. Los registros de salmón se dieron entre marzo y agosto y correspondieron
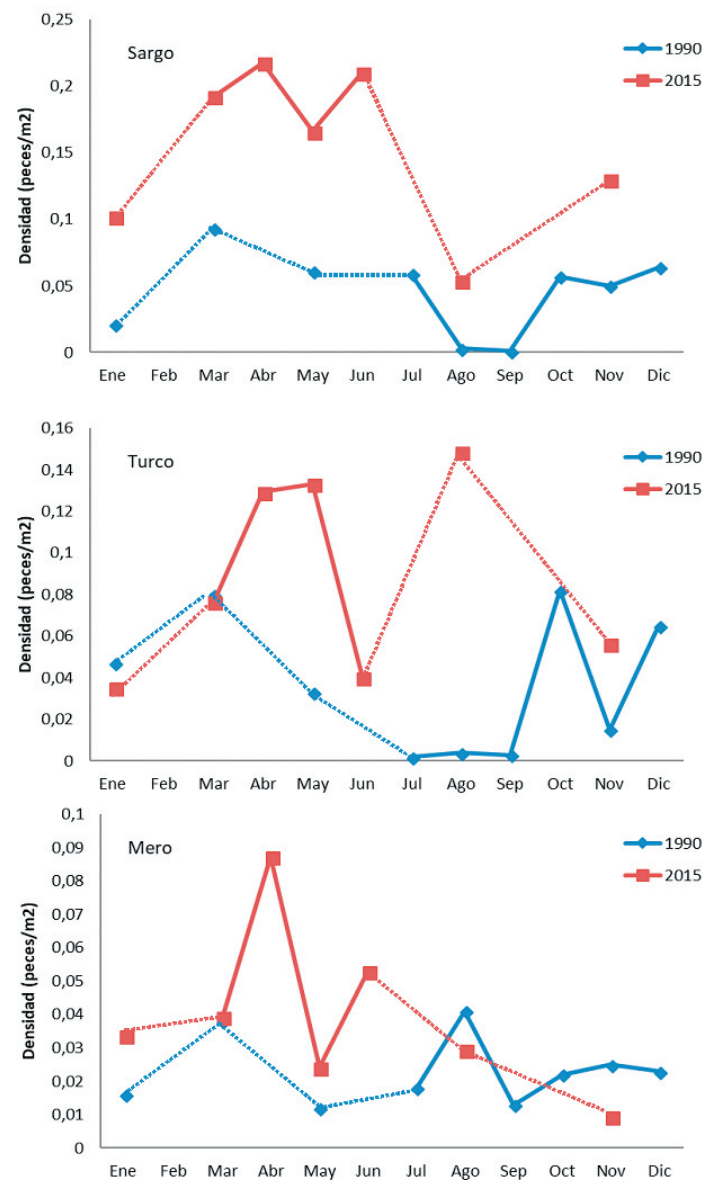

a dos o tres individuos presentes siempre en el mismo sector del PSLG.

Al comparar los resultados obtenidos en 2015 con los resultados de los datos colectados en 1990 (González 1993), se aprecia un aumento general en la abundancia de las especies frecuentes en el PSLG (Figura 4). Este aumento es más evidente en especies como el sargo y el cocherito, que a lo largo del año mantienen el mismo patrón que tenían en 1990, pero con un incremento significativo en su abundancia: se duplicó en el caso del sargo y se triplicó en el caso del cocherito (Figura 4). El turco presentó un patrón contrario al registrado en 1990; se observaron abundancias más altas durante los meses más fríos, con valores que superan hasta seis veces las abundancias de 1990. En el período estival, las abundancias fueron similares a las registradas en 1990 (Figura 4). El salmón también fue más abundante que en 1990; alcanzó el máximo en agosto, a diferencia del muestreo anterior, en el cual el máximo se registró en marzo. Asimismo, en ambos muestreos, los salmones observados corresponden a pocos individuos que viven en un determinado sector del parque y que
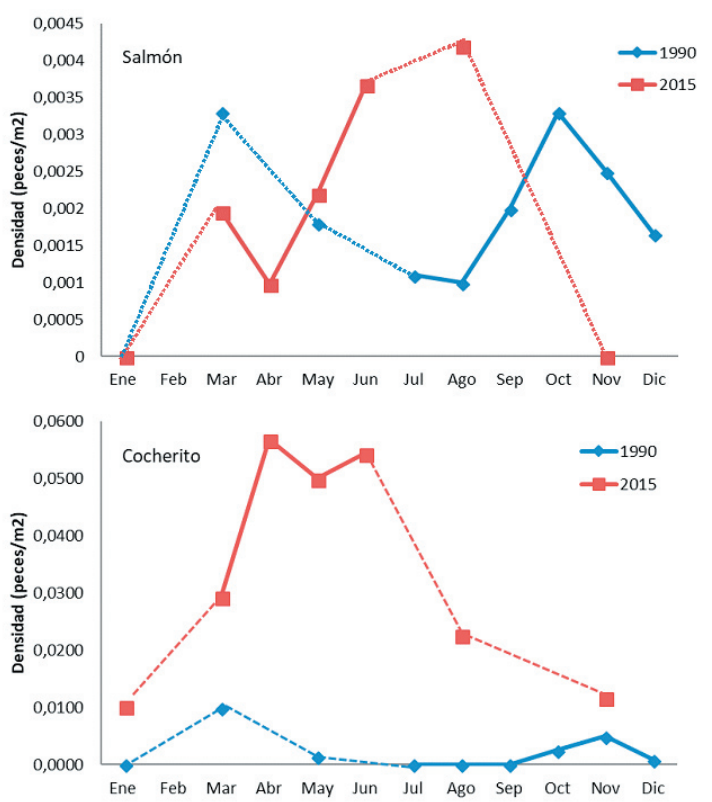

Figura 4. Densidad media de peces en 1990 y 2015, para cada una de las especies presentes en el Parque Submarino Las Grutas.

Figure 4. Mean estimated density of each observed species in the Parque Submarino Las Grutas in 1990 and 2015. 


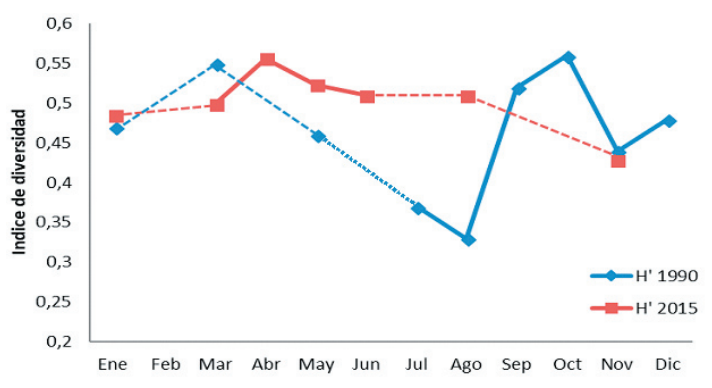

Figura 5. Diversidad de Shannon-Weaver para los meses muestreados en 1990 y 2015.

Figure 5. Shannon-Weaver diversity index for the months sampled in 1990 and 2015.

Tabla 2. Resultados del análisis PERMANOVA de dos vías para poner a prueba diferencias en la composición del ensamble de peces del Parque Submarino Las Grutas entre AÑOS (1990 vs. 2015).

Table 2. PERMANOVA analysis testing differences in species composition of the fish assemblage of Parque Submarino Las Grutas between YEARS (1990 vs. 2015).

\begin{tabular}{lcccc}
\hline $\begin{array}{l}\text { Fuente de } \\
\text { variación }\end{array}$ & df & MS & Pseudo-F & $P$-valor \\
\hline $\begin{array}{l}\text { AÑOS } \\
\text { Res }\end{array}$ & 1 & 683.76 & 7.11 & $<0.001^{*}$ \\
\hline
\end{tabular}

Tabla 3. Resultados del análisis SIMPER mostrando las especies de peces que más contribuyeron a la disimilitud en la composición de especies entre AÑOS (1990 vs. 2015).

Table 3. SIMPER analysis showing the fish species that contributed most to the dissimilarity in fish composition between YEARS (1990 vs. 2015).

\begin{tabular}{lcccc}
\hline & $\begin{array}{c}\text { Disimilitud } \\
\text { promedio }\end{array}$ & $\begin{array}{c}\text { DS } \\
\text { Disimilitud }\end{array}$ & $\begin{array}{c}\text { Contribución } \\
(\%)\end{array}$ & $\begin{array}{c}\text { Acumulado } \\
(\%)\end{array}$ \\
\hline Cocherito & 8.06 & 1.84 & 34.85 & 34.85 \\
Sargo & 5.70 & 1.29 & 24.65 & 59.50 \\
Turco & 4.67 & 1.28 & 20.21 & 79.71 \\
Salmón & 2.64 & 0.96 & 11.43 & 91.14 \\
\hline
\end{tabular}

DS: desviación estándar.

desaparecen en el período estival. Por su parte, el mero mostró un patrón diferente al de 1990, sin registrarse cambios significativos en su abundancia (Figura 4).

El análisis PERMANOVA indicó diferencias significativas para el factor AÑO (Tabla 2). En base a este resultado, se realizó un análisis

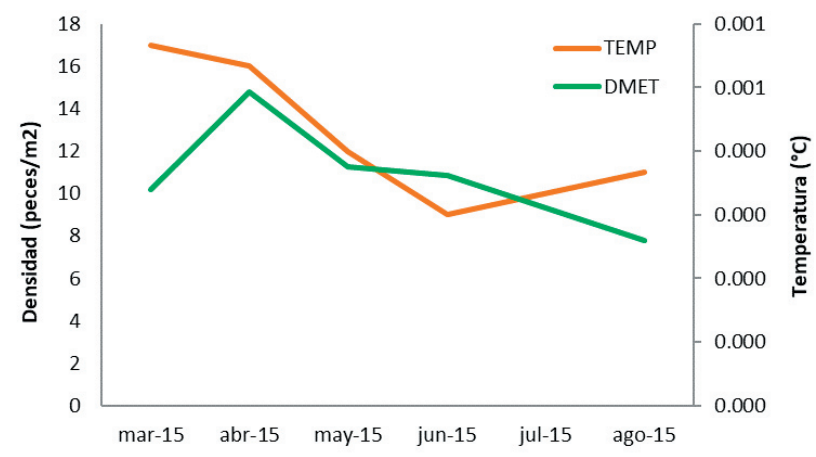

SIMPER y se encontró que las especies que más contribuyeron a la disimilitud entre 1990 y 2015 fueron las especies más abundantes (Tabla 3).

Los valores de diversidad se mantuvieron relativamente estables a lo largo del año en 2015, a diferencia de lo ocurrido en 1990, cuando se detectó una disminución en el valor del índice durante los meses más fríos (Figura 5).

$\mathrm{Al}$ analizar la relación entre la variación en la densidad total de peces en el PSLG y la variación en la temperatura del agua (sobre la base de datos tomados in situ), se observa que, a pesar de que el análisis de correlación fue no significativo (correlación de Pearson, $r=0.47, P=0.46$ ), la mayor abundancia de peces se registró durante los meses más cálidos, con una tendencia decreciente durante los meses más fríos (Figura 6).

\section{Discusión}

En el Mar Argentino son escasos los estudios llevados a cabo in situ sobre peces de arrecifes rocosos (e.g., González 1993; Irigoyen et al. 2003, 2013; Galván et al. 2005, 2009a; Irigoyen 2006; Venerus 2006; Vanella et al. 2007; Venerus et al. 2008, 2014). En gran parte, esto puede deberse a la dificultad para realizar las observaciones, ya que dependen de censos visuales a partir de la práctica de buceo autónomo o de algún sensor visual remoto (e.g., ROV, del inglés remotely operated vehicle). A esta limitante se suman las condiciones meteorológicas adversas del mar patagónico que dificultan o incluso impiden las salidas a muestrear, ya sea por el oleaje, el viento o la mala visibilidad del agua. Todo esto lleva a que se posea un conocimiento limitado de la estructura comunitaria de los peces de arrecifes rocosos de la Patagonia y su variación a lo largo del tiempo.

En el presente estudio se evaluó la variación en la abundancia y diversidad de las especies

Figura 6. Relación entre la abundancia total de peces (eje primario) y la temperatura del agua (eje secundario) (correlación de Pearson, $\mathrm{r}=0.43, P=0.46$ ). TEMP: temperatura del fondo tomada in situ, DMET: densidad media estimada total.

Figure 6. Relationship between total fish abundance (primary axis) and water temperature (secondary axis) (Pearson correlation, $\mathrm{r}=0.47, P<0.46)$. TEMP: bottom temperature taken in situ, DMET: total estimated mean density. 
de peces de arrecifes presentes en el PSLG a lo largo de un ciclo anual y se compararon estos resultados con los obtenidos por González (1993) en 1990, a fin de detectar cambios en la composición del arrecife tras más de 20 años de haber sido sometido a cambios en el ambiente, tanto naturales como producto del impacto antrópico. Los resultados obtenidos indican un aumento en la abundancia de los peces de arrecifes en el PSLG respecto a las abundancias registradas en 1990. Las especies encontradas en la totalidad de los muestreos corresponden a especies templado-cálidas, a excepción de una única observación, la de un individuo de Congiopodus peruvianus (al igual que en el estudio de González [1993]). Esta especie es característica de aguas templadofrías y habita desde el sur de Brasil hasta el extremo sur de América (Menni et al. 1984). Sin embargo, considerando que vive en refugios y que se mimetiza muy bien con el ambiente, es posible que su abundancia y su frecuencia de ocurrencia hayan sido subestimadas en los muestreos.

Las especies templado-cálidas típicas de la provincia biogeográfica Argentina registradas en el presente estudio (i.e., sargo, turco, cocherito, mero y salmón) se corresponden, en general, con lo esperado para los rangos de distribución de dichas especies (Menni and Gostonyi 1982; Menni and López 1984; Cousseau and Perrotta 2000; Balech and Ehrlich 2008). Por otro lado, la menor riqueza de especies templado-frías típicas de la provincia biogeográfica Magallánica ya había sido mencionada por González (1993), por Galván (2008) y Galván et al. (2009a) y por Irigoyen et al. (2013), quienes encontraron un patrón similar para el GSM, el Golfo San José (GSJ) y el Golfo Nuevo (GN). Esta escasez de representantes de la fauna templado-fría puede deberse a que en el área de ecotono estas especies se encontrarían a mayores profundidades, lejos de los sistemas costeros, comparado con la fauna cálido-templada (López 1963; Galván 2008; Galván et al. 2009a). La fauna templado-fría estaría asociada a aguas más frías y densas, con influencia de la corriente de Malvinas y la corriente Patagónica Costera, que corren bajo las aguas más cálidas con influencia de la corriente de Brasil (Boltovskoy et al. 1999; Figueroa et al. 2005; Galván 2008; Galván et al. 2009a). Se ha mencionado que la corriente de Malvinas lleva hacia el norte no sólo aguas subantárticas sino también un bioma de invertebrados y vertebrados que le es propio (Balech and Ehrlich 2008). Por otro lado, en la zona noroeste del GSM donde se llevó a cabo el presente estudio se encuentra la Bahía de San Antonio, una planicie de marea poco profunda con una superficie total de $\sim 127.7 \mathrm{~km}^{2}$, con mareas semidiurnas y un sistema de canales expuestos a fuertes corrientes de marea. Durante los meses de verano, esta planicie de marea tiene un efecto de calentamiento de las aguas aledañas, lo que provoca una variación anual de temperatura de entre 6 y $28^{\circ} \mathrm{C}$. Este efecto de calentamiento de las aguas aledañas podría estar favoreciendo la presencia de especies templado-cálidas en el PSLG.

Siguiendo con el patrón latitudinal en el reemplazo de especies, entre las especies templado-cálidas, el sargo fue la especie más abundante observada en el PSLG. Esta especie fue poco registrada por Galván (2008) y Galván et al. (2009a) en arrecifes rocosos, tanto en el Golfo San José como en el Golfo Nuevo. De forma similar, el cocherito, que estuvo presente en todos los muestreos, fue raramente registrado por Galván (2008) en el GSJ y nunca observado en el GN (Galván et al. 2009; Irigoyen et al. 2013). A diferencia, Galván (2008) y Galván et al. (2009a) registraron un número creciente de especies asociadas con ambientes templados-fríos hacia el sur. Estos resultados son coherentes con el remplazo de componentes subtropicales por templados en el sentido norte-sur.

De las demás especies templado-cálidas registradas en el presente estudio, el turco tuvo abundancias similares a las registradas por Galván (2008) y Galván et al. (2009a) para el GSJ y GN, y tanto el mero como el salmón fueron más abundantes en el GSJ y GN que en el PSLG (Galván et al. 2009a; Venerus et al. 2014). Esto se podría deber a una diferencia en el relieve de los arrecifes entre los tres sitios, ya que la abundancia del mero estaría limitada por la disponibilidad de refugios y la abundancia del salmón por las características de las cavidades que forman los refugios y por el requerimiento de un territorio propio (Galván 2008; Galván et al. 2009a; Venerus et al. 2009, 2014). Los arrecifes rocosos del GSJ y del GN exhiben una fisiografía con mayor cantidad de rocas de gran tamaño y pedregullos, lo que aporta una mayor cantidad y variedad de refugios y microhábitats esenciales para la presencia de especies como el mero y el salmón.

En relación con la abundancia y la diversidad a lo largo del ciclo anual, el ensamble de peces del PSLG mostró variaciones estacionales en 
su composición, vinculadas a la variación en la temperatura. Todas las especies mostraron una abundancia mayor a fines de la temporada cálida, entre marzo y mayo, en correspondencia con el período de reclutamiento de juveniles después de la puesta ocurrida durante los meses de verano. Esta tendencia fue notoria en el caso del sargo, especie de la cual se registraron grandes cardúmenes de juveniles alrededor del arrecife a lo largo de esos meses. Por su parte, el cocherito también mostró un aumento marcado en su abundancia durante el otoño (abril-junio). Las variaciones estacionales en la abundancia de peces de arrecifes rocosos en Patagonia ya habían sido descriptas previamente para el GSJ y el GN (Galván et al. 2009a; Irigoyen et al. 2013; Venerus et al. 2008, 2014).

Si bien el conjunto íctico registrado en el presente estudio fue similar al observado por González (1993), se evidenció un aumento en la abundancia de la totalidad de las especies. En el caso particular del sargo y del cocherito, sus abundancias se duplicaron y triplicaron, respectivamente. Las posibles causas del incremento en la abundancia podrían estar relacionadas con cambios en el medio, ya sea por un aumento de la temperatura del agua o por un cambio en la disponibilidad de alimento.

Los registros de temperatura tomados manualmente in situ durante el período de estudio fueron más altos que los registrados por González (1993) hace 25 años (Figura 7). Los datos de temperatura superficial del agua obtenidos en base a imágenes satelitales desde el año 1985 hasta la actualidad también presentan una tendencia ascendente, con un incremento de $0.8{ }^{\circ} \mathrm{C}$ durante el período considerado (Figura 8). Esta tendencia hacia un calentamiento de la capa superior de los océanos $(0-700 \mathrm{~m})$ se viene registrando a nivel global desde 1980, con un incremento significativo a partir de 1998. Desde 1992 hasta 2015, el contenido de calor de la capa superior de los océanos mostró una tendencia al calentamiento cuatro veces más fuerte que en el período 1960-1992; el 59\% de esta acumulación de calor corresponde a los océanos Atlántico y Glaciar Ártico, que juntos representan sólo 48\% de la superficie de los océanos (Cheng et al. 2017). En los últimos 30 años, el efecto invernadero generado por la concentración de gases en la atmósfera elevó $0.2^{\circ} \mathrm{C}$ la temperatura promedio global. La mayor parte de esta energía calórica es absorbida por los océanos del mundo (HoeghGuldberg and Bruno 2010).

La relación identificada en el presente estudio entre el aumento de la temperatura del agua y el incremento en la abundancia de especies templado-cálidas coincide con lo reportado en otros estudios sobre cambios en la distribución y la abundancia de especies en función de variaciones en las condiciones de los océanos. Perry et al. (2005), en su estudio en el Mar del Norte, reportaron que en los últimos 25 años, dos tercios de las especies de peces comerciales y no comerciales migraron hacia el norte como consecuencia del calentamiento del agua por el cambio climático. Hawkins et al. (2003) y Stebbing et al. (2002), trabajando en el Canal de la Mancha y en la costa de Cornwall, respectivamente, encontraron una relación marcada entre los cambios de temperatura generados por la Oscilación del Atlántico Norte y la migración de especies. En los períodos cálidos, con una duración de entre 20 y 30 años, se produjo un aumento en la abundancia de especies de aguas cálidas que migraron desde el sur, mientras que en los períodos fríos se dio un aumento en la abundancia de especies de aguas frías provenientes del norte. Ambos

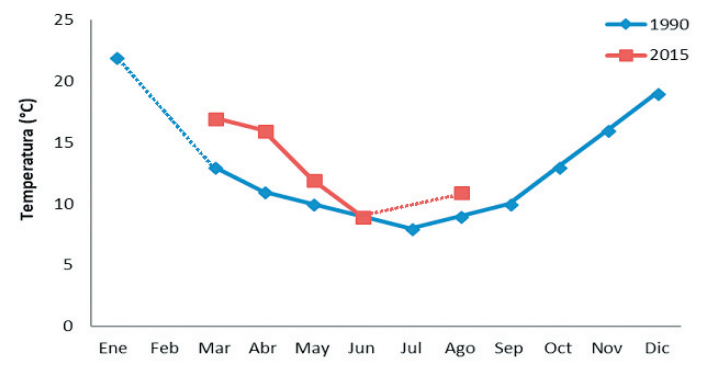

Figura 7. Temperatura del agua de fondo en el Parque Submarino Las Grutas en base a datos tomados in situ. Los datos de 1990 fueron extraídos de González (1993).

Figure 7. Bottom water temperature at the Parque Submarino Las Grutas based on data taken in situ. Data from 1990 were extracted from Gonzalez (1993).

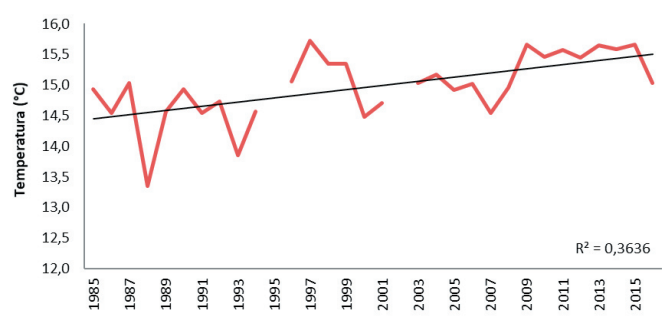

Figura 8. Temperatura superficial del mar para el área del Golfo San Matías. Datos obtenidos de NOAA-NASA.

Figure 8. Sea surface temperature for the San Matías Gulf area. Data obtained from NOAA-NASA. 
estudios coinciden en que en los últimos años hubo una tendencia constante y exponencial hacia el calentamiento de las aguas de la región, por lo que se espera un aumento en la cantidad y la abundancia de especies propias de aguas cálidas. Por su parte, Simpson (2011) menciona que en el Mar del Norte, en el Mar Céltico y en la Bahía de Vizcaya hay una respuesta al calentamiento del agua en el $72 \%$ de las especies de peces estudiadas, y que mayormente esta respuesta implica un incremento en su abundancia. Estos resultados sugieren que el aumento observado en la abundancia de peces de aguas cálidas en el PSLG podría asociarse con un desplazamiento hacia el sur en el límite de la distribución de especies típicas de aguas más cálidas como consecuencia del incremento en la temperatura de las capas superficiales del agua.

Por otro lado, Simpson (2011) menciona que las especies de menor tamaño son las que más fácilmente experimentan un incremento en su abundancia en respuesta al calentamiento del agua. Por su parte, Perry et al. (2005) explican que las especies que migran en respuesta a un incremento en la temperatura del agua tienen ciclos de vida más cortos, son de menor tamaño y poseen una maduración rápida alcanzada con su pequeño porte. Esto coincide con lo registrado en el presente estudio, ya que las especies que registraron un mayor incremento en su abundancia fueron el sargo y el cocherito, ambas de menor porte y ciclos de vida más cortos comparados con el mero y el salmón, que no mostraron cambios significativos en su abundancia. Asimismo, en el Mar del Norte también se registró un aumento marcado en la abundancia de las doradas de mar, Sparus aurata, pertenecientes a la familia Sparidae (misma a la cual pertenece el sargo); se considera que esta es una de las principales familias que responden al aumento de la temperatura del agua con un aumento en su abundancia (Hawkins et al. 2003).

Los cambios en la temperatura podrían estar generando un aumento en la abundancia de ciertas especies no sólo como consecuencia de cambios en la distribución en respuesta a una mejor adaptación fisiológica de ciertas especies de peces, sino también como resultado de un incremento en la producción primaria. Nagelkerken y Connell (2015), en su revisión de 632 publicaciones sobre la alteración de los ecosistemas marinos por la creciente concentración de $\mathrm{CO}_{2}$, mencionan que la producción primaria del plancton no calcificante de aguas templadas se ve favorecida por el aumento de la temperatura y por la mayor concentración de $\mathrm{CO}_{2}$. No obstante, a gran escala, este aumento en la producción primaria no se traduce necesariamente en una mayor producción secundaria. Esto se debe a que una reducción en el pH como consecuencia del aumento en la concentración de $\mathrm{CO}_{2}$ impone costos energéticos sobre el equilibrio ácido-base de los organismos, lo que puede actuar como factor de estrés en especies carnívoras. Lewandowska et al. (2014), al trabajar con modelos de circulación global, también sostienen que el aumento de la temperatura tiene un efecto positivo en la abundancia del fitoplancton, pero que esto puede ser contrarrestado por los efectos negativos generados en el flujo de nutrientes.

Además de la temperatura, otra posible causa de un aumento en la producción primaria es el aporte de nutrientes de origen antrópico, lo cual podría conducir a cambios en la abundancia de ciertas especies de peces. Este problema, denominado eutrofización marina costera, es el proceso de enriquecimiento de las aguas a partir del aporte externo de nutrientes, principalmente nitrógeno y fósforo. El enriquecimiento estimula la producción primaria y puede generar floraciones algales visibles (Santinelli 2008). El primer efecto de la eutrofización es un aumento de la producción de fitoplancton, lo que se puede traducir en un incremento en la cantidad de individuos, a pesar de una disminución en la diversidad debido a la dominancia de ciertas especies (Santinelli 2008). Como ya se mencionó antes, la eutrofización es un fenómeno estudiado y descripto en el área de estudio, que muestra una tendencia creciente durante los últimos años dado el aumento de la población local y el turismo en la región (Santinelli 2008; Martinetto et al. 2010; Teichberg et al. 2010). En particular en el interior de la Bahía de San Antonio, Martinetto et al. (2010) observaron una elevada abundancia y diversidad de consumidores (invertebrados pequeños y aves) asociados con las áreas con mayores tasas de eutrofización. Los autores sugieren que la gran descarga de agua durante el ciclo de las mareas podría prevenir eventos anóxicos o hipóxicos, lo que haría que el ambiente resultara adecuado para los consumidores. De esta forma, la Bahía de San Antonio y su área de influencia podría ser un caso en el que la eutrofización soporta altas densidades de consumidores mediante el aumento de la disponibilidad de alimentos, en lugar de afectar negativamente a la supervivencia de los organismos. Este efecto podría, a su vez, 
conducir a un aumento de la abundancia de algunas especies de arrecifes, principalmente de especies omnívoras como el sargo y el turco. Estas especies exhiben un alto solapamiento en su nicho trófico, depredando sobre organismos que habitan los arrecifes (e.g., erizos de mar, lapas, bivalvos, cangrejos, poliquetos, entre otros) (Galván et al. 2009b; Funes et al. 2014). Desafortunadamente, no se disponen de series temporales de abundancia y disponibilidad de dichas presas como para evaluar de forma empírica la relación entre el cambio observado en la abundancia de peces y la disponibilidad de alimento.

Como los procesos que ocurren en un ecosistema no son aislados y dependen unos de otros se debe tener en cuenta el efecto en conjunto de los diferentes factores que afectan a la producción primaria. La temperatura ascendente combinada con incrementos en la carga de fósforo y nitrógeno fomentan el crecimiento y la acumulación de biomasa de una comunidad natural de fitoplancton (De Senerpont Domis et al. 2014). Si se tiene en cuenta lo antes mencionado, es probable que tanto la temperatura como el aporte externo de nutrientes tengan un efecto sinérgico y que generen en conjunto un aumento de la producción primaria que, a su vez, podría reflejarse en un incremento en la abundancia de algunas especies de peces de arrecife en el PSLG. Al presente no se dispone de series temporales de productividad primaria para el área que nos permitan evaluar la asociación entre dicha variable ambiental y los datos biológicos.

Los resultados obtenidos en el marco del presente estudio reafirman la importancia de disponer de bases de datos que permitan monitorear el estado de los ecosistemas costeros patagónicos a largo plazo. Esta información es esencial a fin de establecer medidas de manejo y conservación que garanticen la preservación de la estructura y funcionamiento de los ecosistemas bajo una mirada holística del sistema.

Agradecimientos. Los autores agradecen a la Empresa Cota Cero - Las Grutas, Río Negro - Argentina por brindar el soporte logístico para llevar a cabo los muestreos. El estudio fue realizado gracias al financiamiento de la Agencia Nacional de Promoción Científica y Tecnológica (PICT 2014-1671) y al aporte de una Beca Estímulo a la Vocación Científica del Consejo Interuniversitario Nacional.

\section{REFERENCIAS}

Anderson, M. J. 2001. A new method for non-parametric multivariate analysis of variance. Austral Ecology 26:32-46. Aubone, A. 2000. El colapso de la merluza (Merluccius hubbsi) y su recuperación biológica. INIDEP Informe Técnico 17:1-22.

Balech, E., and M. D. Ehrlich. 2008. Esquema Biogeográfico del Mar Argentino. Revista de Investigación y Desarrollo Pesquero 19:45-75

Boltovskoy, D., M. J. Gibbons, L. Hutchings, and D. Binet. 1999. General biological features of the South Atlantic. South Atlantic Zooplankton. Backhuys Publishers, Leiden.

Burnham, K. P., D. R. Anderson, and J. L. Laake. 1985. Efficiency and bias in strip and line transect sampling. The Journal of Wildlife Management 49(4):1012-1018.

Castro, P., and M. E. Huber. 2007. Biología Marina. 6ta edición. McGraw-Hill Interamericana, Madrid, España.

Cheng, L., K. E. Trenberth, J. Fasullo, T. Boyer, J. Abraham, and J. Zhu. 2017. Improved estimates of ocean heat content from 1960 to 2015. Science Advances 3(3):e1601545.

Clarke K. R. 1993. Non-parametric multivariate analyses of changes in community structure. Australian Journal of Ecology 18:117-143.

Clarke, K., and R. Warwick. 2001. Change in marine communities: An approach to statistical analysis and interpretation. PRIMER-E Ltd., Plymouth. Pp. 82.

Cousseau, M. B., and R. G. Perrotta. 2000. Peces marinos de Argentina: biología, distribución, pesca (Segunda Edición). INIDEP, Mar del Plata, Argentina.

De Senerpont Domis, L. N., D. B. Van de Waal, N. R. Helmsing, E. Van Donk, and W. Mooij. 2014. Community stoichiometry in a changing world: combined effects of warming and eutrophication on phytoplankton dynamics. Ecology 95(6):1485-1495.

Di Giácomo, E. E., J. Calvo, M. R. Perier, and E. Morriconi. 1993. Spawning aggregations of Merluccius hubbsi, in Patagonian waters: evidence for a single stock? Fisheries Research 16(1):9-16.

Dias, J. A., M. M. D. Mahiques, and A. Cearreta. 2012. Coastal Management: the result of a doubtful relationship between Man and Nature. Revista de Gestão Costeira Integrada 12:3-6.

Elías, I., and C. Rajoy. 1992. Hábitos alimenticios del salmón de mar Pseudopercis semifasciata (Cuvier, 18929): Pinguipedidae, en aguas norpatagónicas argentinas. Revista de Biología Marina 27:133-146.

Figueroa, D. E., J. M. Díaz de Astarloa, and R. Reta. 2005. Unusual finding of Paranotothenia magellanica (Nototheniidae) on the temperate north coast of Argentina. Cybium 29(1):97-99. 
Funes, M., A. L. Liberoff, and D. E. Galván. 2014. Cambios tamaño-dependientes en la dieta de peces marinos y su estudio mediante análisis de isótopos estables. Ecología Austral 24(1):118-126.

Galván, D. E. 2008. Ensambles de peces en los arrecifes norpatagónicos: diversidad, abundancia y relaciones tróficas y con el hábitat. Tesis doctoral. Centro Regional Bariloche, Universidad Nacional del Comahue, Río Negro. Pp. 147.

Galván, D. E., L. A. Venerus, A. J. Irigoyen, A. M. Parma, and A. E. Gosztonyi. 2005. Extension of the distributional range of the silver porgy, Diplodus argenteus (Valenciennes 1830), and the red porgy, Pagrus pagrus (Linnaeus 1758) (Sparidae) in northern Patagonia, south'western Atlantic. Journal of Applied Ichthyology 21(5):444-447.

Galván, D. E., L. A. Venerus, and A. J. Irigoyen. 2009a. The Reef-fish Fauna of the Northern Patagonian Gulfs, Argentina, Southwestern Atlantic. The Open Fish Science Journal 2:90-98.

Galván, D. E., F. Botto, A. M. Parma, L. Bandieri, N. Mohamed, and O. O. Iribarne. 2009b. Food partitioning and spatial subsidy in shelter-limited fishes inhabiting patchy reefs of Patagonia. Journal of Fish Biology 75:2585-2605.

González, R. A. 1993. Variaciones en la abundancia de las especies ícticas durante un ciclo anual, en una restinga del submareal costero norpatagónico. Actas de las Jornadas Nacionales de Ciencias del Mar (1991). Pp. 118-128.

González, R. A. 1998. Biología y explotación pesquera del salmón de mar Pseudopercis semifasciata (Cuvier, 1829) (Pinguipedidae) en el golfo San Matías, Argentina. Tesis doctoral. Universidad Nacional del Sur, Buenos Aires, Argentina. Pp. 135.

González, R. A., M. A. Narvarte, and G. M. Caille. 2007. An assessment of the sustainability of the hake Merluccius hubbsi artisanal fishery in San Matías Gulf, Patagonia, Argentina. Fisheries Research 87(1):58-67.

Hawkins, S. J., A. J. Southward, and M. J. Genner. 2003. Detection of environmental change in a marine ecosystem evidence from the western English Channel. Science of the Total Environment 310(1):245-256.

Hoegh-Guldberg, O., and J. F. Bruno. 2010. The impact of climate change on the world's marine ecosystems. Science 328(5985):1523-1528.

Hollebone, A. L., and M. E. Hay. 2008. An invasive crab alters interaction webs in a marine community. Biological Invasions 10(3):1314-1318.

Instituto Nacional de Estadística y Censos (INDEC). 1991. Instituto Geográfico Nacional (IGN). URL: goo.gl/ nz88wB.

Instituto Nacional de Estadística y Censos (INDEC). 2010. Instituto Geográfico Nacional (IGN). URL: goo.gl/DzVerc.

Irigoyen, A. J. 2006. Distribución espacial y temporal del mero (Acanthistius brasilianus) en la plataforma argentina. Tesis de Licenciatura, Universidad Nacional de la Patagonia San Juan Bosco, Puerto Madryn. Pp. 54.

Irigoyen, A. J., D. E. Galván, and L. A. Venerus. 2003. Registro de dos especies de peces de aguas cálido-templadas, Epinephelus marginatus (Lowe 1834) y Seriola lalandi Valenciennes 1833, en los golfos norpatagónicos. Libro de Resúmenes de las V Jornadas Nacionales de Ciencias del Mar, 123.

Irigoyen, A. J., D. E. Galván, L. A. Venerus, and A. M. Parma. 2013. Variability in abundance of temperate reef fishes estimated by visual census. PLoS ONE 8:e61072.

Jackson, J. B. C., M. X. Krby, W. H. Berger, K. A. Bjorndal, L. W. Botsford, B. J. Bourque, R. H. Bradbury, R. Cooke, J. Erlandson, J. A. Estes, T. P. Hughes, S. Kidwell, C. B. Lange, H. S. Lenihan, J. M. Pandolfi, C. H. Peterson, R. S. Steneck, M. J. Tegner, and R. R. Warner. 2001. Historical overfishing and the recent collapse of coastal ecosystems. Science 293:629-637.

Johnston, E. L., and D. A. Roberts. 2009. Contaminants reduce the richness and evenness of marine communities: A review and meta-analysis. Enviromental Pollution 157:1745-1752.

Johnson, C. R., S. C. Banks, N. S. Barrett, F. Cazassus, P. K. Dunstan, G. J. Edgar, S. D. Frusher, C. Gardner, M. Haddon, F. Helidoniotis, K. L. Hill, N. J. Holbrook, G. W. Hosie, P. R. Last, S. D. Ling, J. Melbourne-Thomas, K. Miller, G. T. Pecl, A. J. Richardson, K. R. Ridgway, S. R. Rintoul, D. A. Ritz, D. JeffRoss, J. Craig Sanderson, S. A. Shepherd, A. Slotwinski, K. M. Swadling, and N. Taw. 2011. Climate change cascades: Shifts in oceanography, species' ranges and subtidal marine community dynamics in eastern Tasmania. Journal of Experimental Marine Biology and Ecology 400(1):17-32.

Josefson, A. B., J. L. S. Hansesn, G. Asmund, and P. Johansen. 2008. Threshold response of benthic macrofauna integrity to metal contamination in West Greenland. Marine Pollution Bulletin 56:1265-1274.

Kaiser, M. J., and S. J. De Groot. 2001. Effects of fishing on non-target species and habitats: biological, conservation and socio-economic issues. Blackwell Science, Oxford, UK.

Kokot, R. R., and F. Chomnalez. 2012. Retroceso de la línea de costa en las Grutas, provincia de Río Negro. Revista de las Asociación Geológica Argentina 69:88-96.

Lewandowska, A. M., D. G. Boyce, M. Hofmann, B. Matthiessen, U. Sommer, and B. Worm. 2014. Effects of sea surface warming on marine plankton. Ecology Letters 17(5):614-623.

López, R. B. 1963. Problemas sobre la distribución geográfica de los peces marinos sudamericanos. Revista del Museo Argentino de Ciencias Naturales, Buenos Aires, Argentina. Hidrobiología 1:111-135.

Magurran, A. E. 1988. Why diversity? Ecological diversity and its measurement. Springer Netherlands, Dordrecht.

Martinetto, P., P. Daleo, M. Escapa, J. Alberti, J. P. Isacch, E. Fanjul, et al. 2010. High abundance and diversity of consumers associated with eutrophic areas in a semi-desert macrotidal coastal ecosystem in Patagonia, Argentina. Estuarine, Coastal and Shelf Science 88:357-364.

Menni, R. C., and A. E. Gosztonyi. 1982. Benthic and semidemersal fish associations in the Argentine Sea. Studies on Neotropical Fauna and Environment 17(1):1-29. 
Menni, R. C., and H. L. López. 1984. Distributional patterns of Argentine marine fishes. Physis, Sección A. 42:71-85.

Menni, R. C., R. A. Ringuelet, and R. H. Aramburu. 1984. Peces marinos de la Argentina y Uruguay. Editorial Hemisferio Sur S. A., Buenos Aires, Argentina.

Menni, R., and M. Stehmann. 2000. Distribution, environment and biology of batoid fishes off Argentina, Uruguay and Brazil. A review. Revista del Museo Argentino de Ciencias Naturales Nueva Serie 2(1):69-109.

Millán, D. 2015. Anuario de Estadísticas Pesqueras de la Provincia de Río Negro. Dpto. Policía de Pesca, Dirección de Pesca.

Moreno, C. E. 2001. Métodos para medir la biodiversidad. M\&T-Manuales y Tesis SEA, vol.1., Zaragoza, España.

Nagelkerken, I., and S. D. Connell. 2015. Global alteration of ocean ecosystem functioning due to increasing human CO2 emissions. Proceedings of the National Academy of Sciences 112(43):13272-13277.

Narvarte, M. A. 2006. Plan de Monitoreo de Contaminación de la zona costera del Golfo San Matías. Informe Final. Proyecto Prevención de la Contaminación Costera y Gestión de la Diversidad Biológica Marina. Donación GEF ${ }^{\circ}$ 28385 AR. Secretaría de Ambiente y Desarrollo Sustentable. Pp. 18.

Oksanen, J., F. G. Blanchet, M. Friendly, R. Kindt, P. Legendre, D. McGlinn, P. R. Minchin, R. B. O'Hara, G. L. Simpson, P. Solymos, M. H. H. Stevens, E. Szoecs, and H. Wagner. 2017. vegan: Community Ecology Package. R package version 2.4-5. URL: goo.gl/WSr3dw.

Perry, A. L., P. J. Low, J. R. Ellis, and J. D. Reynolds. 2005. Climate change and distribution shifts in marine fishes. Science 308(5730):1912-1915.

Pielou, E. C. 1975. Ecological diversity. Wiley, New York, Estados Unidos.

Piola, A. R., and L. M. Scasso. 1988. Circulación en el golfo San Matías. Geoacta 15(1):33-51.

Romero, M. A., R. A. González, and M. Ocampo-Reinaldo. 2008. Patrón temporal en la composición específica de los desembarcos de la pesquería de arrastre demersal del Golfo San Matías. IBMP Serie Publicaciones 7:27-37.

Romero, M. A., R. A. González, and M. Ocampo-Reinaldo. 2010. When conventional fisheries management measures are not effective to reduce the catch and discard of juvenile fish: a case study of Argentine hake trawl fishery in San Matías Gulf (Patagonia, Argentina). North American Journal of Fisheries Management 30:702-712.

Rubinich, J. P. 2000. Edad y crecimiento del mero Acanthistius brasilianus (Pisces, Serranidae) en el Golfo San Matías. Tesis de grado. Universidad Nacional de la Patagonia San Juan Bosco. Chubut, Argentina. Pp. 33.

Sadovy, Y., and W. L. Cheung. 2003. Near extinction of a highly fecund fish: trouble among the croakers. Fish and Fisheries 4:86-99.

Sanabra, F. 2002. Buceando recuerdos-Matices de la historia del buceo en la Argentina. Ocean Ediciones Submarinas, Puerto Madryn, Argentina.

Santinelli, N. H. 2008. Fitoplancton de un ambiente costero sometido a perturbación antrópica: Bahía Nueva, Provincia de Chubut. Tesis doctoral. Facultad de Ciencias Naturales de la Universidad Nacional de la Patagonia San Juan Bosco. Sede Trelew, Chubut, Argentina. Pp. 217.

Scalise, A., E. Schnack, E. Fucks, K. Ahrendt, R. González, F. García, R. Sciarrone, and D. Spagnuolo. 2009. Evaluación de alternativas para la conservación y manejo del frente costero en Las Grutas. Consejo Federal de Inversiones, San Antonio Oeste, Argentina.

Simpson, S. D., S. Jennings, M. P. Johnson, J. L. Blanchard, P. J. Schön, D. W. Sims, and M. J. Genner. 2011. Continental shelf-wide response of a fish assemblage to rapid warming of the sea. Current Biology 21(18):1565-1570.

Stebbing, A. R. D., S. M. T. Turk, A. Wheeler, and K. R. Clarke. 2002. Immigration of southern fish species to southwest England linked to warming of the North Atlantic (1960-2001). Journal of the Marine Biological Association of the UK 82(02):177-180.

Stige, L. C., G. Ottersen, K. Brander, K. S. Chan, and N. C. Stenseth. 2006. Cod and climate: effect of the North Atlantic Oscillation on recruitment in the North Atlantic. Marine Ecology Progress Series 325:227-241.

Teichberg, M., S. E. Fox, Y. S. Olsen, I. Valiela, P. Martinetto, et al. 2010. Eutrophication and macroalgal blooms in temperate and tropical coastal waters: nutrient enrichment experiments with Ulva spp. Global Change Biology 16(9): 2624-2637.

Vanella, F. A., D. A. Fernández, M. C. Romero, and J. Calvo. 2007. Changes in the fish fauna associated with a subAntarctic Macrocystis pyrifera kelp forest in response to canopy removal. Polar Biology 30(4):449-457.

Venerus, L. A. 2006. Dinámica espacial del salmón de mar Pseudopercis semifasciata (Cuvier, 1829) (Pinguipedidae). Implicancias para el uso sustentable de los sistemas de arrecifes rocosos. Tesis doctoral. Facultad de Ciencias Exactas y Naturales de la Universidad de Buenos Aires, Buenos Aires, Argentina. Pp. 191.

Venerus, L. A., A. M. Parma, and D. E. Galván. 2008. Annual occupation pattern of temperate rocky reefs by the Argentine sandperch Pseudopercis semifasciata in San José Gulf Marine Park, Argentina. Fisheries Management and Ecology 15:217-229.

Venerus, L. A., A. J. Irigoyen, D. E. Galván, and A. M. Parma. 2014. Spatial dynamics of the Argentine sandperch, Pseudopercis semifasciata (Pinguipedidae), in temperate rocky reefs from northern Patagonia, Argentina. Marine and Freshwater Research 65:39-49. 